\title{
BOUNDS FOR PROJECTION CONSTANTS AND 1-SUMMING NORMS
}

\author{
HERMANN KÖNIG AND NICOLE TOMCZAK-JAEGERMANN
}

\begin{abstract}
It is shown that projection constants $\lambda\left(X_{n}\right)$ of $n$-dimensional normed spaces $X_{n}$ satisfy $\lambda\left(X_{n}\right) \leq \sqrt{n}-c / \sqrt{n}$ where $c>0$ is a numerical constant. Similarly, the 1-summing norms of (the identity of) $X_{n}$ can be estimated by $\pi_{1}\left(X_{n}\right) \geq \sqrt{n}+c / \sqrt{n}$. These estimates are the best possible: for prime $n$, translation-invariant $n$-dimensional spaces $X_{n}$ such that $\lambda\left(X_{n}\right) \geq \sqrt{n}-2 / \sqrt{n}$ and $\pi_{1}\left(X_{n}\right) \leq \sqrt{n}+2 / \sqrt{n}$ can be constructed. For these spaces Gordon-Lewis constants and distances to Hilbert spaces are large as well: $\operatorname{gl}\left(X_{n}\right) \geq \frac{1}{3} \sqrt{n}, d\left(X_{n}, l_{2}^{n}\right)=\sqrt{n}$.
\end{abstract}

\section{INTRODUCTION AND RESULTS}

The main results of the paper complete the investigation of an upper bound for the projection constants of arbitrary finite-dimensional Banach spaces, which had started with the Kadec-Snobar [KaS] estimate: $\lambda\left(X_{n}\right) \leq \sqrt{n}$, for any $n$ dimensional space $X_{n}$. This estimate was further studied in several papers [KöLL, Kö, KöL, Lew2]. In particular, it has recently been shown by Lewis [Lew2] that $\lambda\left(X_{n}\right) \leq \sqrt{n}-n^{-3 / 2} 5^{-2(2 n+1)}$. Our main theorem gives an essential strengthening, stated in the abstract.

Upper bounds for projection constants are intimately connected to lower bounds for the 1-summing norm of the identity operator. For spaces with enough symmetries these bounds follow from each other by a simple formal argument. In general, it turns out that similar but somewhat simpler calculations than for the projection constants lead to the lower bound for $\pi_{1}\left(X_{n}\right)$.

Both upper and lower bounds are the best possible, up to terms of higher order. The approach used by König in [Kö], based on some number theoretic results, gives the relevant constructions. The same approach leads to a construction of a finite-dimensional space with large Gordon-Lewis constant. This provides the first deterministic example of such a space.

Our notation essentially follows [TJ]. For completeness, let us recall the main definitions.

By $\mathbf{K}$ we denote the scalar field, either the real numbers $\mathbf{R}$ or the complex numbers $\mathbf{C}$.

Received by the editors May 31, 1988 and, in revised form, October 12, 1988.

1980 Mathematics Subject Classification (1985 Revision). Primary 47B10, 46B20.

The first author was partially supported by Deutsche Forschungsgemeinschaft and NSERC Operating Grant.

The second author was partially supported by NSERC Operating Grant. 
The relative projection constant of a (closed) subspace $X$ of a Banach space $Y$ is defined by

$$
\lambda(X, Y):=\inf \{\|P\| \mid P: Y \rightarrow X \subset Y \text { is a linear projection onto } X\} ;
$$

the (absolute) projection constant of a Banach space $X$ is given by

$$
\lambda(X):=\sup \{\lambda(X, Y) \mid Y \text { is a Banach space containing } X \text { as a subspace }\} \text {. }
$$

Let $1 \leq p \leq \infty$ and $u: Y \rightarrow Z$ be a (continuous linear) operator between Banach spaces. The p-factorization norm of $u$ is defined as

$$
\begin{aligned}
& \gamma_{p}(u):=\inf \{\|v\|\|w\| \mid \exists \text { measure space }(\Omega, \mu) \text { and operators } \\
& \left.\qquad v: Y \rightarrow L_{p}(\Omega, \mu) \text { and } w: L_{p}(\Omega, \mu) \rightarrow Z \text { with } u=w v\right\},
\end{aligned}
$$

all these expressions being possibly $\infty$. Any separable Banach space $X$ can be isometrically embedded into $l_{\infty}$. Thus

$$
\lambda(X)=\lambda\left(X, l_{\infty}\right)=\gamma_{\infty}\left(\operatorname{Id}_{X}\right)
$$

where $\operatorname{Id}_{X}$ denotes the identity map on $X$. For $1 \leq p<\infty$ and $u: Y \rightarrow Z$ the p-summing norm $\pi_{p}(u)$ is the infimum over all $c>0$ such that

$$
\left(\sum_{i=1}^{n}\left\|u y_{i}\right\|^{p}\right)^{1 / p} \leq c \sup _{\left\|y^{*}\right\|_{Y^{*}=1}}\left(\sum_{i=1}^{n}\left|\left\langle y^{*}, y_{i}\right\rangle\right|^{p}\right)^{1 / p}
$$

holds for all finite sequences $y_{1}, \ldots, y_{n} \in Y$. We set $\pi_{p}(u)=\infty$ if no such $c$ exists. We let $\pi_{p}(X)=\pi_{p}\left(\operatorname{Id}_{X}\right)$. It is well known that $\gamma_{\infty}$ and $\pi_{1}$ are in trace-duality; i.e., for any finite-dimensional spaces $Y$ and $Z$ and any operator $u: Y \rightarrow Z$

$$
\gamma_{\infty}(u)=\sup _{0 \neq v: Z \rightarrow Y} \frac{|\operatorname{tr}(v u)|}{\pi_{1}(v)} \text { and } \pi_{1}(u)=\sup _{0 \neq v: Z \rightarrow Y} \frac{|\operatorname{tr}(v u)|}{\gamma_{\infty}(v)} .
$$

In particular,

$$
\lambda(X)=\sup _{0 \neq v: X \rightarrow X} \frac{|\operatorname{tr}(v)|}{\pi_{1}(v)} .
$$

This implies that for an $n$-dimensional space $X$ we get

$$
\lambda(X) \pi_{1}(X) \geq n .
$$

A space $X$ is said to have enough symmetries if any operator $u: X \rightarrow X$ which commutes with all isometries of $X$ is a multiple of the identity map. If $X$ has enough symmetries and $\operatorname{dim} X=n$, then

$$
\lambda(X) \pi_{1}(X)=n ;
$$

i.e., the sup in (1) is attained by $v=\mathrm{Id}_{X}$ [GG]. We also need the Gordon-Lewis constant of $X$,

$$
\operatorname{gl}(X):=\sup \left\{\gamma_{1}(u) / \pi_{1}(u) \mid 0 \neq u: X \rightarrow l_{2}\right\},
$$


and the Banach-Mazur distance from $X$ to $Y$,

$$
d(X, Y):=\inf \left\{\|u\|\left\|u^{-1}\right\| \mid u: X \rightarrow Y \text { is an isomorphism }\right\} .
$$

The unconditional basis constant $\mathrm{ubc}\left(x_{j}\right)$ of a basis $\left(x_{j}\right)$ of $X$ is the smallest constant $c>0$ such that for all $\left(a_{j}\right) \subseteq \mathbf{K}$ and $\left(\varepsilon_{j}\right) \subseteq \mathbf{K}$ with $\left|\varepsilon_{j}\right|=1$,

$$
\left\|\sum_{j} \varepsilon_{j} a_{j} x_{j}\right\| \leq c\left\|\sum_{j} a_{j} x_{j}\right\| .
$$

One has $\operatorname{gl}(X) \leq \operatorname{ubc}\left(x_{j}\right)$ for any basis $\left(x_{j}\right)$ of $X$ and $\operatorname{ubc}(X):=\inf \left\{\operatorname{ubc}\left(x_{j}\right) \mid\right.$ $\left(x_{j}\right)$ a basis of $\left.X\right\} \leq(\operatorname{dim} X)^{1 / 2}$ (cf., e.g., [TJ, §34]).

If a complex space $X$ is a translation-invariat subspace of the $L_{\infty}$-space on a compact commutative group and $\left(y_{j}\right)$ is the natural basis of characters in $X$, then the Sidon constant of $X, S(X)$, is the smallest constant $c>0$ such that for all $\left(a_{j}\right) \subset \mathbf{C}$,

$$
\sum_{j}\left|a_{j}\right| \leq c\left\|\sum_{j} a_{j} y_{j}\right\| .
$$

It can be shown that $S(X)=\operatorname{ubc}\left(y_{j}\right) \leq(\operatorname{dim} X)^{1 / 2}$ (cf. [Pis], also the proof of Proposition 5).

Our main result for the projection constant states

Theorem 1. For all $n \in \mathbf{N}$ and any $n$-dimensional space $X_{n}$ we have

$$
\lambda\left(X_{n}\right) \leq \sqrt{n}-1 / 2 \sqrt{n}+O\left(1 / n^{3 / 4}\right) .
$$

Remark 1. For real 2-dimensional spaces $X_{2}^{\mathbf{R}}$ the estimates yield $\lambda\left(X_{2}^{\mathbf{R}}\right) \leq$ 1.378 , while the conjectured extreme value is $\frac{4}{3}$.

We use superscripts $\mathbf{R}$ and $\mathbf{C}$ to distinguish between slightly different estimates in the real and complex cases. A general estimate for the relative projection constant of an $n$-dimensinal subspace $X_{n}$ of a $N$-dimensional space $Y_{N}$ was given in [KöLL]. It states

$$
\begin{aligned}
\lambda\left(X_{n}, Y_{N}\right) & \leq f(n, N):=\sqrt{n}\left(\frac{\sqrt{n}+\sqrt{N-1} \sqrt{N-n}}{N}\right) \\
& \leq \sqrt{n}\left(1-\frac{(\sqrt{n}-1)^{2}}{2 N}\right) .
\end{aligned}
$$

In the case when $X_{n}$ has enough symmetries, Theorem 1 can be slightly improved.

Proposition 2. Let $X_{n}$ have enough symmetries or (at least) assume that $\pi_{1}\left(X_{n}\right) \lambda\left(X_{n}\right)=n$. Then

$$
\begin{aligned}
& \lambda\left(X_{n}^{\mathbf{R}}\right) \leq f\left(n, \frac{n(n+1)}{2}\right)=\sqrt{n}-\frac{1}{\sqrt{n}}+\frac{2}{n}-O\left(\frac{1}{n^{3 / 2}}\right), \\
& \lambda\left(X_{n}^{\mathbf{C}}\right) \leq f\left(n, n^{2}\right)=\sqrt{n}-\frac{1}{2 \sqrt{n}}+\frac{1}{n}-O\left(\frac{1}{n^{3 / 2}}\right) .
\end{aligned}
$$


Remark 2. For 2- and 3-dimensional real spaces with enough symmetries, $\lambda\left(X_{2}^{\mathbf{R}}\right) \leq \frac{4}{3}$ and $\lambda\left(X_{3}^{\mathbf{R}}\right) \leq(1+\sqrt{5}) / 2$. Equality holds for the spaces with the hexagon unit ball and the dodecahedron unit ball, respectively. The $\pi_{1}$-norm is minimal for these spaces.

Conjecture. We conjecture that the estimates of Proposition 2 are true in general (without assuming enough symmetries).

It is well known that $\pi_{2}\left(X_{n}\right)=\sqrt{n}$ [GG], cf. also [TJ, §9]. This yields an obvious inequality $\sqrt{n}=\pi_{2}\left(X_{n}\right) \leq \pi_{1}\left(X_{n}\right)$. Techniques of Theorem 1 and Proposition 2 yield the following lower bound for $\pi_{1}\left(X_{n}\right)$ (without assuming enough symmetries).

Proposition 3. For all $n \in \mathbf{N}$ and any $n$-dimensional space $X_{n}$ we have

$$
\begin{aligned}
& \pi_{1}\left(X_{n}^{\mathbf{R}}\right) \geq n / f\left(n, \frac{n(n+1)}{2}\right)=\sqrt{n}+\frac{1}{\sqrt{n}}-\frac{2}{n}+O\left(\frac{1}{n^{3 / 2}}\right), \\
& \pi_{1}\left(X_{n}^{\mathbf{C}}\right) \geq n / f\left(n, n^{2}\right)=\sqrt{n}+\frac{1}{2 \sqrt{n}}-\frac{1}{n}+O\left(\frac{1}{n^{3 / 2}}\right) .
\end{aligned}
$$

Remark 3. The method also works for $\pi_{p}\left(X_{n}\right)$ if $1 \leq p<2$, yielding $\pi_{p}\left(X_{n}\right) \geq$ $\sqrt{n}+c_{p} / \sqrt{n}$. Neglecting terms of higher order $O(1 / n), c_{p}$ may be taken as $2\left((1 / p)-\frac{1}{2}\right)$ in the real case and $(1 / p)-\frac{1}{2}$ in the complex case.

The estimates in Theorem 1 and Propositions 2 and 3 are very close to being the best possible, in view of the following constructions.

Proposition 4. (a) For any prime $p$ and $m \in \mathbf{N}$, set $n:=p^{m}+1$ and $N:=$ $n^{2}-n+1$. One can construct a complex $n$-dimensional space $X_{\infty}^{n} \subset l_{\infty}^{N}$ with $\lambda\left(X_{\infty}^{n}\right) \pi_{1}\left(X_{\infty}^{n}\right)=n$ such that

$$
\lambda\left(X_{\infty}^{n}\right)=f(n, N)=\sqrt{n}-\frac{1}{2 \sqrt{n}}+\frac{1}{n}-O\left(\frac{1}{n^{3 / 2}}\right)
$$

and

$$
\pi_{1}\left(X_{\infty}^{n}\right)=n / f(n, N)=\sqrt{n}+\frac{1}{2 \sqrt{n}}-\frac{1}{n}+O\left(\frac{1}{n^{3 / 2}}\right) .
$$

(b) For any $n=4^{m}$ and $N:=n^{2} / 2$ one can construct a real $n$-dimensional space $X_{\infty}^{n} \subset l_{\infty}^{N}$ with

$$
\sqrt{n}-2 / \sqrt{n} \leq \lambda\left(X_{\infty}^{n}\right) \leq \sqrt{n}-1 / \sqrt{n} .
$$

Thus in (a) the difference between the complex example and the general estimate of Propositions 2 and 3 is only of order

$$
f\left(n, n^{2}\right)-f\left(n, n^{2}-n+1\right)=O\left(1 / n^{3 / 2}\right) .
$$

The spaces constructed in (a) are translation invariant over finite groups. Another version of this construction leads to spaces with many more "almost extremal" properties. In particular, they would have the gl-constants of maximal order and extremal distance to Hilbert spaces. Moreover, if $\left(y_{j}\right)_{j=1}^{n}$ is the natural basis of characters in $X_{\infty}^{n}$, then $\operatorname{ubc}\left(y_{j}\right)=\sqrt{n}$ or, equivalently, the Sidon constant of $X_{\infty}^{n}$ is extremal and equal to $\sqrt{n}$. 
Proposition 5. For all cdd prime numbers $n$ and $N:=\left(n^{2}-1\right) / 2$ one can construct complex $n$-dimensional translation-invariant spaces $X_{\infty}^{n} \subset l_{\infty}^{N}$ with

(a) $\lambda\left(X_{n}\right)=\sqrt{n}-2 / \sqrt{n}-O(1 / n)$,

(b) $\pi_{1}\left(X_{n}\right)=\sqrt{n}+2 / \sqrt{n}+O(1 / n)$,

(c) the distance to $l_{2}^{n}$ is maximal, $d\left(X_{\infty}^{n}, l_{2}^{n}\right)=\sqrt{n}$,

(d) the natural basis of characters $\left(y_{j}\right)$ in $X_{\infty}^{n}$ satisfies $\operatorname{ubc}\left(y_{j}\right)=\sqrt{n}$,

(e) the Sidon constant is maximal, $S\left(X_{\infty}^{n}\right)=\sqrt{n}$,

(f) the Gordon-Lewis constant is of maximal order $\operatorname{gl}\left(X_{\infty}^{n}\right) \geq \frac{1}{3} \sqrt{n}$.

Remark 4. For any space $X_{n}$ one has $\operatorname{gl}\left(X_{n}\right) \leq \sup \left\{\lambda\left(Y_{n}\right) \mid \operatorname{dim} Y_{n}=n\right\} \leq$ $\sqrt{n}-c / \sqrt{n}$.

The remaining part of the paper contains mostly the proofs of statements and remarks and is organized as follows. $\S 2$ contains proofs of general estimates of Theorem 1 and Propositions 2 and 3. In $\S 3$ the constructions of examples are presented and some more related estimates are obtained.

\section{Projection CONSTANTS, SUMming NORMS, AND ULTRASPHERICAL POLYNOMIALS}

Theorem 1 and Propositions 2 and 3 are based on a similar argument, which requires several steps presented below. The "proper" proofs of the stated results are provided at the end of the section.

The differences $\pi_{1}\left(X_{n}\right)-\sqrt{n}$ and $\sqrt{n}-\lambda\left(X_{n}\right)$ will be estimated from below by a suitable double integral $I$, which is then bounded from below. The basic idea of the first step is due to Lewis [Lew2].

Step 1 . Let $X$ be a real or complex $n$-dimensinal space and define $0 \leq \varepsilon<1$ by $\pi_{1}(X)=\sqrt{n} /(1-\varepsilon)$. We want to bound $\varepsilon$ from below (like $\varepsilon \geq c / n$ ) to get a lower estimate for $\pi_{1}(X)$ and, if $\lambda(X)=n / \pi_{1}(X)$ as in Proposition 2, an upper bound for $\lambda(X)$. By Pietsch's factorization theorem (cf., e.g., [LT, TJ, §9]), there exist a compact space $T$ and a regular Borel probability measure $\mu$ on $T$, an isometric embedding $i: X \rightarrow L_{\infty}(T, \mu)$, and a map $w: L_{1}(T, \mu) \rightarrow L_{\infty}(T, \mu)$ with $\|w\|=\pi_{1}(X)=\sqrt{n} /(1-\varepsilon)$ such that the following diagram commutes.

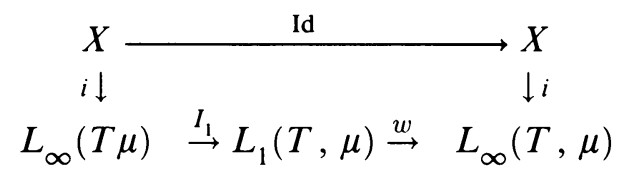

For $1 \leq p<\infty$, denote by $I_{p}$ the inclusion $L_{\infty}(T, \mu) \rightarrow L_{p}(T, \mu)$ and let $X_{\infty}:=i(X)$ and $X_{p}:=I_{p} i(X)$ be the images of $X$ in $L_{\infty}(T, \mu)$ and $L_{p}(T, \mu)$, respectively. Thus for all $g \in X_{\infty}$,

$$
\|g\|_{\infty}=\left\|w I_{1} g\right\|_{\infty} \leq \pi_{1}(X) \int_{T}|g(s)| d \mu(s) .
$$


By Lewis [Lew1], the $n$-dimensional space $X_{1} \subset L_{1}(T, \mu)$ admits a basis $\left(f_{i}\right)_{i=1}^{n} \subset X$ such that

$$
n \int_{T} f_{j}(s) \overline{f_{k}(s)} f(s)^{-1} d \mu(s)=\delta_{j k}
$$

where $f$ is the square function $f(s)=\left(\sum_{j=1}^{n}\left|f_{j}(s)\right|^{2}\right)^{1 / 2}$. (The result in [Lew1] is stated for real spaces only, but the proof works in the complex case, too.)

Let us make a general remark which will be used several times throughout the argument. If $g_{j} \in L_{\infty}(T, \mu)$ for $j=1, \ldots, n$, then, for almost all $s$,

$$
\sum_{j=1}^{n}\left|g_{j}(s)\right|^{2} \leq\left\|\sum_{j=1}^{n} g_{j}(s) \overline{g_{j}}\right\|_{\infty}
$$

Indeed, this estimate is obvious if the $g_{j}$ 's are continuous. The general case easily follows by approximating a measurable function by a continuous function on sets of measure arbitrarily close to 1 .

Let $h_{j}(s):=\sqrt{n} f_{j}(s) / f(s), s \in T$, and make the change of density $d \nu(s)=$ $f(s) d \mu(s)$. Then

$$
\sum_{j=1}^{n}\left|h_{j}(s)\right|^{2}=n \quad \text { and } \quad \int_{T} h_{j}(s) \overline{h_{k}(s)} d \nu(s)=\delta_{j k}
$$

Note that $\nu$ is a probability measure on $T$ as well, since by (5),

$$
\nu(T)=\int_{T} f(s) d \mu(s)=\int_{T}\left(\sum_{j=1}^{n}\left|f_{j}(s)\right|^{2} / f(s)\right) d \mu(s)=1 .
$$

Let $h(s, t):=\sum_{j=1}^{n} h_{j}(s) \overline{h_{j}(t)}$. Using (5), (6), and (4), we find

$$
\begin{aligned}
n & =n\left(\int_{T} f(s) d \mu(s)\right)^{2} \leq n \int_{T}|f(s)|^{2} d \mu(s) \\
& \leq \int_{T}\left\|\sum_{j=1}^{n} n f_{j}(s) \overline{f_{j}}\right\|_{\infty} d \mu(s) \\
& \leq \pi_{1}(X) \int_{T} \int_{T}\left|\sum_{j=1}^{n} n f_{j}(s) \overline{f_{j}(t)}\right| d \mu(t) d \mu(s) \\
& =\pi_{1}(X) \int_{T} \int_{T}|h(s, t)| d \nu(s) d \nu(t) .
\end{aligned}
$$


We choose $\alpha>0$ close to $\sqrt{n}$ and estimate the mean difference of $(\alpha / n)|h(s, t)|$ and 1 using (7) and (8). We have

$$
\begin{aligned}
I: & =\int_{T} \int_{T}\left(\frac{\alpha}{n}|h(s, t)|-1\right)^{2} d \nu(t) d \nu(s) \\
= & \int_{T}\left(\int_{T} \frac{\alpha^{2}}{n^{2}}\left|\sum_{j=1}^{n} h_{j}(s) \overline{h_{j}(t)}\right|^{2} d \nu(t)\right) d \nu(s)+1 \\
& -2 \frac{\alpha}{n} \int_{T} \int_{T}|h(s, t)| d \nu(t) d \nu(s) \\
\leq & \frac{\alpha^{2}}{n}+1-2 \frac{\alpha}{\pi_{1}(X)}=2 \frac{\alpha}{\sqrt{n}} \varepsilon+\left(\frac{\alpha}{\sqrt{n}}-1\right)^{2} .
\end{aligned}
$$

Inequality (9) provides a lower estimate for $\varepsilon$ in terms of $I$. For instance, if $\alpha=\sqrt{n}$, then $\varepsilon \geq \frac{1}{2} I$. However, the final lower bounds for $\varepsilon$, obtained by estimating $I$ from below, will be optimized by taking $\alpha:=\sqrt{n+2}$ for real spaces and $\alpha:=\sqrt{n+1}$ for complex spaces. This will be of interest for small dimensions $n$.

Step 2. Before bounding $I$ from below, we give the more elaborate estimate of type (9) in the case of general projection constants, i.e., in the situation of Theorem 1. For this, define $0 \leq \varepsilon<1$ by $\lambda(X)=\sqrt{n}(1-\varepsilon)$. By (1), there is $v: X \rightarrow X$ such that $\lambda(X)=\operatorname{tr}(v) / \pi_{1}(v)$. We normalize $\pi_{1}(v)=\sqrt{n}$ so that $\operatorname{tr}(v)=n(1-\varepsilon)$. Again by Pietsch's factorization theorem, there exist a compact space $T$ and a regular Borel probability measure $\mu$ on $T$, an isometric embedding $i: X \rightarrow L_{\infty}(T, \mu)$, and an "extension" $w: L_{1}(T, \mu) \rightarrow L_{\infty}(T, \mu)$ of $v$ with $\|w\|=\pi_{1}(v)=\sqrt{n}$ such that the following diagram commutes.

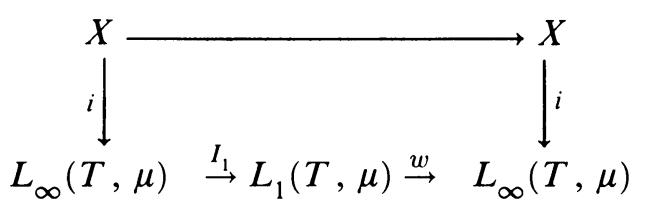

Again, for all $g \in X_{\infty}:=i(X)$ and $\bar{v}:=w I_{1}$, we have

$$
\|\bar{v}(g)\|_{\infty} \leq \pi_{1}(v) \int_{T}|g(s)| d \mu(s) .
$$

The basis $f_{j}$ in $X_{1}$ satisfying (5) and the functions $h_{j}$ and $h$ satisfying (7) are constructed as before. Since $f_{j}$ and $\overline{n f_{j}} / f$ are $\mu$-biorthogonal, using $\left(4^{\prime}\right)$, 
(5), and (6) we find

$$
\begin{aligned}
\operatorname{tr}(v) & =\sum_{j=1}^{n}\left\langle\bar{v}\left(f_{j}\right), n \overline{f_{j}} / f\right\rangle_{\mu}=\int_{T}\left(\sum_{j=1}^{n} n \overline{f_{j}(s)} / f(s) \bar{v}\left(f_{j}\right)(s)\right) d \mu(s) \\
& \leq \int_{T}\left\|\sum_{j=1}^{n} n \overline{f_{j}(s)} / f(s) \bar{v}\left(f_{j}\right)\right\|_{\infty} d \mu(s) \\
& \leq \pi_{1}(v) \int_{T} \int_{T}\left|\sum_{j=1}^{n} n \overline{f_{j}(s)} / f(s) f_{j}(t)\right| d \mu(t) d \mu(s) \\
& =\pi_{1}(v) \int_{T} \int_{T}|h(s, t)| d \nu(t) d \mu(s) .
\end{aligned}
$$

This and (7) imply, for a fixed $\alpha>0$ (chosen later or to be close to $\sqrt{n}$ ),

$$
\begin{aligned}
J & :=\int_{T} \int_{T}\left(\frac{\alpha}{n}|h(s, t)|-1\right)^{2} d \nu(t) d \mu(s) \\
& \leq 2 \frac{\alpha}{\sqrt{n}} \varepsilon+\left(\frac{\alpha}{\sqrt{n}}-1\right)^{2}=: \delta_{1}(\varepsilon, n) .
\end{aligned}
$$

Unfortunately, in contrast to $I$, the integral $J$ is not symmetric. We symmetrize it using the fact that $h$ is hermitian; i.e., $h(s, t)=\overline{h(t, s)}$. We have

$$
\begin{aligned}
\delta_{1}(\varepsilon, n) & \geq J=\int_{T} \int_{T}\left(\frac{\alpha}{n}|h(s, t)|-1\right)^{2} \frac{f(t)+f(s)}{2} d \mu(t) d \mu(s) \\
& \geq \int_{T} \int_{T}\left(\frac{\alpha}{n}|h(s, t)|-1\right)^{2} \sqrt{f(t)} d \mu(t) \sqrt{f(s)} d \mu(s) .
\end{aligned}
$$

In the situation of Theorem 1 we may assume that $\varepsilon<1 / 2 n$, since we want to show that $\varepsilon \geq 1 / 2 n-c / n^{5 / 4}$ for some $c>0$. Define measures $\tilde{\lambda}$ and $\lambda$ on $T$ by $d \tilde{\lambda}(t)=\sqrt{f(t)} d \mu(t), \lambda=\tilde{\lambda} / \tilde{\lambda}(T)$. Thus $\lambda$ is a probability measure on $T$. We claim that

$$
\tilde{\lambda}(T) \geq(1+\sqrt{2 \varepsilon} /(1-\sqrt{2 n \varepsilon}))^{-1}=: \delta_{2}(\varepsilon, n)^{-1},
$$

so that with $\delta(\varepsilon, n):=\delta_{1}(\varepsilon, n) \delta_{2}(\varepsilon, n)^{2}$,

$$
\delta(\varepsilon, n) \geq \int_{T} \int_{T}\left(\frac{\alpha}{n}|h(s, t)|-1\right)^{2} d \lambda(t) d \lambda(s)=: I
$$

as in (9). To estimate $I$ from below (in both (9) and $\left(9^{\prime \prime}\right)$ ), we shall use only the fact that $\nu$ and $\lambda$ are probability measures.

Step 3. To prove (10), it suffices to show that

$$
\|f\|_{L_{2}(T, \mu)} \leq \delta_{2}(\varepsilon, n) .
$$


Indeed, Hölder's inequality yields

$$
1=\|f\|_{L_{1}(T, \mu)}^{3} \leq\|f\|_{L_{2}(T, \mu)}^{2}\|f\|_{L_{1 / 2}(T, \mu)} .
$$

Thus,

$$
\tilde{\lambda}(T)=\int_{T} \sqrt{f(t)} d \mu(t)=\|f\|_{L_{1 / 2}(T, \mu)}^{1 / 2} \geq\|f\|_{L_{2}(T, \mu)}^{-1},
$$

completing the proof of (10).

We first show that $v$, considered as a map on $X_{2} \subset L_{2}(T, \mu)$, differs not too much from $\operatorname{Id}_{X}$. Let $j: L_{2}(T, \mu) \rightarrow L_{1}(T, \mu)$ denote the inclusion map. Then $\tilde{v}:=I_{2} w j: L_{2}(T, \mu) \rightarrow L_{2}(T, \mu)$ actually maps $X_{2}$ into itself. We restrict our attention to $\tilde{v}: X_{2} \rightarrow X_{2}$. Since for an operator $S$ acting in a Hilbert space,

$$
\operatorname{tr}\left(S S^{*}\right)=\operatorname{hs}(S)^{2}=\pi_{2}(S)^{2},
$$

where $\mathrm{hs}(S)$ is the Hilbert-Schmidt norm of $S$, and since $\pi_{2}\left(I_{2}\right)=1$ (cf., e.g., [TJ, §10]), we have

$$
\begin{aligned}
\pi_{2}\left(\operatorname{Id}-\tilde{v}: X_{2} \rightarrow X_{2}\right)^{2} & =\operatorname{tr}\left((\operatorname{Id}-\tilde{v})\left(\operatorname{Id}-\tilde{v}^{*}\right): X_{2} \rightarrow X_{2}\right) \\
& =n-2 \operatorname{tr}\left(\tilde{v}: X_{2} \rightarrow X_{2}\right)+\operatorname{tr}\left(\tilde{v} \tilde{v}^{*}: X_{2} \rightarrow X_{2}\right) \\
& \leq n-2 \operatorname{tr}(v)+\pi_{2}\left(\tilde{v}: X_{2} \rightarrow X_{2}\right)^{2} \\
& \leq n-2 n(1-\varepsilon)+\left(\pi_{2}\left(I_{2}\right)\|w\|\right)^{2}=2 n \varepsilon .
\end{aligned}
$$

Hence

$$
\left\|\mathrm{Id}-\tilde{v}: X_{2} \rightarrow X_{2}\right\| \leq \sqrt{2 n \varepsilon}<1 .
$$

Thus $\tilde{v}$ is invertible on $X_{2}$ and

$$
\left\|\tilde{v}^{-1}: X_{2} \rightarrow X_{2}\right\| \leq 1 /(1-\sqrt{2 n \varepsilon}) .
$$

To estimate $\|f\|_{L_{2}(T, \mu)}$ write

$$
\begin{aligned}
\|f\|_{L_{2}(T, \mu)} & =\left(\int_{T} \sum_{j=1}^{n}\left|f_{j}(s)\right|^{2} d \mu(s)\right)^{1 / 2} \\
& \leq\left(\int_{T} \sum_{j=1}^{n}\left|\tilde{v} f_{j}(s)\right|^{2} d \mu(s)\right)^{1 / 2}+\left(\int_{T} \sum_{j=1}^{n}\left|(\operatorname{Id}-\bar{v}) f_{j}(s)\right|^{2} d \mu(s)\right)^{1 / 2} .
\end{aligned}
$$


To estimate the first term, we use (6), $\left(4^{\prime}\right)$, and (7), to get the following inequality valid almost everywhere on $T$ :

$$
\begin{aligned}
\sum_{j=1}^{n}\left|\bar{v}\left(f_{j}\right)(s)\right|^{2} & \leq|| \sum_{j=1}^{n} \overline{\bar{v}\left(f_{j}\right)(s)} \bar{v}\left(f_{j}\right) \|_{\infty} \\
& \leq \sqrt{n} \int_{T}\left|\sum_{j=1}^{n} \overline{\bar{v}\left(f_{j}\right)(s)} f_{j}(t)\right| d \mu(t) \\
& =(\sqrt{n} / \sqrt{n}) \int_{T}\left|\sum_{j=1}^{n} \overline{\bar{v}\left(f_{j}\right)(s)} h_{j}(t)\right| d \nu(t) \\
& \leq\left(\int_{T}\left|\sum_{j=1}^{n} \overline{\bar{v}\left(f_{j}\right)(s)} h_{j}(t)\right|^{2} d \nu(t)\right)^{1 / 2} \\
& =\left(\sum_{j=1}^{n}\left|\bar{v}\left(f_{j}\right)(s)\right|^{2}\right)^{1 / 2} .
\end{aligned}
$$

Thus $\sum_{j=1}^{n}\left|\bar{v}\left(f_{j}\right)(s)\right|^{2} \leq 1$ for almost all $s$. To estimate the second term, observe that algebraically, the spaces $X_{\infty}, X_{2}$, and $X_{1}$ coincide and $\bar{v}$ is algebraically the same map as $\tilde{v}$. Using the definition of the $\pi_{2}$-norm we get

$$
\begin{aligned}
& \left(\int_{T} \sum_{j=1}^{n}\left|(\mathrm{Id}-\bar{v}) f_{j}(s)\right|^{2} d \mu(s)\right)^{1 / 2}=\left(\sum_{j=1}^{n}\left\|(\mathrm{Id}-\tilde{v}) f_{j}\right\|^{2}\right)^{1 / 2} \\
& \leq \pi_{2}\left(\mathrm{Id}-\tilde{v}: X_{2} \rightarrow X_{2}\right) \sup _{\|\eta\|_{2}=1}\left(\int_{T}\left|\sum_{j=1}^{n} \eta_{j} f_{j}(s)\right|^{2} d \mu(s)\right)^{1 / 2} .
\end{aligned}
$$

Combining these estimates with (12) and (7) we get

$$
\begin{aligned}
\|f\|_{L^{2}(T, \mu)} & \leq 1+\frac{\sqrt{2 \varepsilon n}}{\sqrt{n}} \sup _{\|\eta\|_{2}=1}\left(\int_{T}\left|\sum_{j=1}^{n} \eta_{j} h_{j}(s)\right|^{2} d \nu(s)\right)^{1 / 2}\|f\|_{\infty}^{1 / 2} \\
& =1+\sqrt{2 \varepsilon\|f\|_{\infty}} .
\end{aligned}
$$

To estimate $\|f\|_{\infty}$, we use the fact that, by (13), $\tilde{v}^{-1}: X_{2} \rightarrow X_{2}$ is well defined, and thus $\bar{v}^{-1}: X_{1} \rightarrow X_{1}$ is well defined too. Hence, by $(6)$ and $\left(4^{\prime}\right)$, we have, 
$\mu$-a.e.,

$$
\begin{aligned}
|f(s)|^{2} & =\sum_{j=1}^{n}\left|f_{j}(s)\right|^{2} \leq\left\|\sum_{j=1}^{n} \overline{f_{j}(s)} \bar{v} \bar{v}^{-1} f_{j}\right\|_{\infty} \\
& \leq \sqrt{n} \int_{T}\left|\sum_{j=1}^{n} \overline{f_{j}(s)} \bar{v}^{-1} f_{j}(t)\right| d \mu(t) \\
& \left.\leq \sqrt{n}\left(\int_{T}\left|\sum_{j=1}^{n} \overline{f_{j}(s)} \tilde{v}^{-1} f_{j}(t)\right|^{2} d \mu(t)\right)^{1 / 2}\right)^{1 / 2} \\
& \leq\left.\left.\sqrt{n}\left\|\tilde{v}^{-1}: X_{2} \rightarrow X_{2}\right\|\left|\int_{T}\right| \sum_{j=1}^{n} \overline{f_{j}(s)} f_{j}(t)\right|^{2} d \mu(t)\right|^{1 / 2} \\
& \leq \sqrt{n}\left\|\tilde{v}^{-1}\right\|\left(\|f\|_{\infty}^{1 / 2} / \sqrt{n}\right)\left(\int_{T}\left|\sum_{j=1}^{n} \overline{f_{j}(s)} h_{j}(t)\right|^{2} d \nu(t)\right)^{3 / 2} \\
& =\left\|\tilde{v}^{-1}\right\| \cdot\|f\|_{\infty}^{3 / 2}
\end{aligned}
$$

This yields $\|f\|_{\infty}^{1 / 2} \leq\left\|\tilde{v}^{-1}\right\|$ and thus, by (13) and (14),

$$
\|f\|_{L_{2}(T, \mu)} \leq 1+\sqrt{2 \varepsilon} /(1-\sqrt{2 n \varepsilon})=\delta_{2}(\varepsilon, n) .
$$

We thus proved (11), (10), and $\left(9^{\prime \prime}\right)$.

Step 4. We now turn to estimating the double integral

$$
I:=\int_{T} \int_{T}\left(\frac{\alpha}{n}|h(s, t)|-1\right)^{2} d \lambda(s) d \lambda(t)
$$

from below, for arbitrary probability measure $\lambda$ on $T$. The following facts on ultraspherical and Jacobi polynomials can be found in Müller [M], Levenštein [Lev], and Kabatyanskii-Levenštein [KaL].

Let $S^{n-1}(\mathbf{K})=\left\{\left.x \in \mathbf{K}^{n}|| x\right|_{2}=1\right\}$. For all $k, n \in \mathbf{N}$ there are polynomials $R_{k}^{n}$ of degree $k$ and functions $w_{k i}^{n}: S^{n-1}(\mathbf{K}) \rightarrow \mathbf{K}, i=1, \ldots, r_{k}^{n}$, such that for all $x, y \in S^{n-1}(\mathbf{K})$ the following "addition formula" for $R_{k}^{n}$ holds:

$$
R_{k}^{n}\left(|\langle x, y\rangle|^{2}\right)=\sum_{i=1}^{r_{k}^{n}} w_{k i}^{n}(x) \overline{w_{k i}^{n}(y)} .
$$

These functions differ in the real and complex cases. In terms of Jacobi polynomials $P_{k}^{\alpha, \beta}$, which are orthogonal polynomials on $[-1,1]$ with weight function $(1-v)^{\alpha}(1+v)^{\beta}$, one has

$$
\begin{aligned}
& R_{k}^{n}(v)=c_{n k} P_{k}^{(n-3) / 2,-1 / 2}(2 v-1), \quad \mathbf{K}=\mathbf{R} \text { and } v \in[0,1], \\
& R_{k}^{n}(v)=c_{n k} P_{k}^{n-2,0}(2 v-1), \quad \mathbf{K}=\mathbf{C} \text { and } v \in[0,1] .
\end{aligned}
$$


For $k=0,1,2$ we have, with a suitable normalization,

$$
\begin{aligned}
& \left.\begin{array}{l}
R_{0}^{n}(v)=1 \\
R_{1}^{n}(v)=n v-1
\end{array}\right\}, \quad \mathbf{K}=\mathbf{R} \text { or } \mathbf{C}, \\
& R_{2}^{n}(v)=(n+2)(n+4) v^{2}-6(n+2) v+3, \quad \mathbf{K}=\mathbf{R}, \\
& R_{2}^{n}(v)=(n+1)(n+2) v^{2}-4(n+1) v+2, \quad \mathbf{K}=\mathbf{C} .
\end{aligned}
$$

The functions $w_{k i}^{n}$ are harmonic polynomials which are homogeneous of degree $2 k$ in $x$ in the real case and homogeneous of degrees $k$ in $x$ and $\bar{x}$ in the complex case; $r_{k}^{n}$ are the dimensions of these spaces of polynomials,

$$
\begin{aligned}
& r_{k}^{n}=\left(\begin{array}{c}
n+2 k-1 \\
n-1
\end{array}\right)-\left(\begin{array}{c}
n+2 k-3 \\
n-1
\end{array}\right), \quad \mathbf{K}=\mathbf{R}, \\
& r_{k}^{n}=\left(\begin{array}{c}
n+k-1 \\
n-1
\end{array}\right)^{2}-\left(\begin{array}{c}
n+k-2 \\
n-1
\end{array}\right), \quad \mathbf{K}=\mathbf{C},
\end{aligned}
$$

but we will not use the last facts. The use of (15) to estimate $I$ was inspired by Levenštein's bounds for the number of balls in spherical packing problems.

For $s \in T$, let $x_{s}:=(1 / \sqrt{n})\left(h_{j}(s)\right)_{j=1}^{n} \in \mathbf{K}^{n}$. By (7), $\left|x_{s}\right|_{2}=1$ for almost all $s$. Since $\left|\left\langle x_{s}, x_{t}\right\rangle\right| \leq 1$, we find

$$
\left(\frac{\alpha}{n}|h(s, t)|-1\right)^{2}=\left(\alpha\left|\left\langle x_{s}, x_{t}\right\rangle\right|-1\right)^{2} \geq \frac{1}{(\alpha+1)^{2}}\left(\alpha^{2}\left|\left\langle x_{s}, x_{t}\right\rangle\right|^{2}-1\right)^{2} .
$$

Set $F(v):=\left(\alpha^{2} v-1\right)^{2} /(\alpha+1)^{2}$, for $v \in[0,1]$. Then double integral $I$ is bounded from below by

$$
I \geq \int_{T} \int_{T} F\left(\left|\left\langle x_{s}, x_{t}\right\rangle\right|^{2}\right) d \lambda(s) d \lambda(t) .
$$

An easy calculation shows, using (16), that

$$
F(v)=f_{0}^{n}+f_{1}^{n} R_{1}^{n}(v)+f_{2}^{n} R_{2}^{n}(v),
$$

where

$$
\begin{aligned}
& f_{0}^{n}=\frac{1}{(\alpha+1)^{2}}\left(1-\frac{2 \alpha^{2}}{n}+\frac{3 \alpha^{4}}{n(n+2)}\right), \\
& f_{1}^{n}=\frac{1}{(\alpha+1)^{2}}\left(\frac{6 \alpha^{4}}{n(n+4)}-\frac{2 \alpha^{2}}{n}\right), \\
& f_{2}^{n}=\frac{1}{(\alpha+1)^{2}} \frac{\alpha^{4}}{(n+2)(n+4)} \text { if } \mathbf{K}=\mathbf{R} ; \\
& f_{0}^{n}=\frac{1}{(\alpha+1)^{2}}\left(1-\frac{2 \alpha^{2}}{n}+\frac{2 \alpha^{4}}{n(n+1)}\right), \\
& f_{1}^{n}=\frac{1}{(\alpha+1)^{2}}\left(\frac{4 \alpha^{4}}{n(n+2)}-\frac{2 \alpha^{2}}{n}\right), \\
& f_{2}^{n}=\frac{1}{(\alpha+1)^{2}} \frac{\alpha^{4}}{(n+1)(n+2)} \quad \text { if } \mathbf{K}=\mathbf{C} .
\end{aligned}
$$


Hence $f_{k}^{n} \geq 0$ if $\alpha \geq \sqrt{(n+4) / 3}$ if $\mathbf{K}=\mathbf{R}$ or $\alpha \geq \sqrt{(n+2) / 2}$ if $\mathbf{K}=\mathbf{C}$ for $k=0,1,2$. Using this, (15) and (17), and the fact that $\lambda$ is a probability measure on $T$, we get

$$
\begin{aligned}
I & \geq f_{0}^{n}+\sum_{k=1}^{2} f_{k}^{n} \int_{T} \int_{T} R_{k}^{n}\left(\left|\left\langle x_{s}, x_{t}\right\rangle\right|^{2}\right) d \lambda(s) d \lambda(t) \\
& =f_{0}^{n}+\sum_{k=1}^{2} f_{k}^{n} \sum_{i=1}^{r_{k}}\left|\int_{T} w_{k i}^{n}\left(x_{s}\right) d \lambda(s)\right|^{2} \geq f_{0}^{n} .
\end{aligned}
$$

Now we are prepared for short proofs of our results. Roughly speaking, they are based on the fact that for $\alpha \sim \sqrt{n}, f_{0}^{n}$ is of order $1 / n$. More delicate calculations show much more precise estimates.

Proof of Theorem 1. Let $\lambda(X)=\sqrt{n}(1-\varepsilon)$. If $\varepsilon \geq 1 /(2 n)$, the conclusion is obviously satisfied. Assume that $\varepsilon<1 /(2 n)$. By $\left(9^{\prime \prime}\right)$, (18), and (19) we get

$$
\delta(\varepsilon, n)=2 \varepsilon\left(1+\frac{\sqrt{2 \varepsilon}}{1-\sqrt{2 \varepsilon n}}\right)^{2} \geq I \geq f_{0}^{n} .
$$

Putting $c_{n}:=\sqrt{f_{0}^{n}}$ and $\delta=\sqrt{2 \varepsilon}$, this means

$$
\delta^{2}-\frac{1+c_{n} \sqrt{n}}{\sqrt{n}-1} \delta+\frac{c_{n}}{\sqrt{n}-1} \leq 0
$$

and thus

$$
\sqrt{2 \varepsilon}=\delta \geq \frac{1}{2(\sqrt{n}-1)}\left\{\left(1+c_{n} \sqrt{n}\right)-\sqrt{\left(1-c_{n} \sqrt{n}\right)^{2}+4 c_{n}}\right\}=: \delta_{n} .
$$

Thus $\varepsilon \geq c / n$ for some $c>0$. Asymptotically, in the real case

$$
1-c_{n} \sqrt{n}=(\sqrt{2}-1)+O(1 / \sqrt{n}), \quad \delta_{n}=1 / \sqrt{n}-O(1 / n),
$$

and hence

$$
\varepsilon \geq 1 / 2 n-O\left(1 / n^{3 / 2}\right) .
$$

In the complex case the estimate is slightly worse; namely,

$$
1-c_{n} \sqrt{n}=O(1 / \sqrt{n}), \quad \delta_{n}=1 / \sqrt{n}-O\left(1 / n^{3 / 4}\right),
$$

and hence

$$
\varepsilon \geq 1 / 2 n-O\left(1 / n^{5 / 4}\right) .
$$

In both cases we get the bound

$$
\lambda(X) \leq \sqrt{n}(1-\varepsilon) \leq \sqrt{n}-1 / 2 \sqrt{n}+O\left(1 / n^{3 / 4}\right) .
$$

This completes the proof.

In the case of 2-dimensional real spaces, the choice of $\alpha=\frac{3}{2}$ leads to the numerical bound $\lambda\left(X_{2}^{\mathbf{R}}\right) \leq 1.378$ given in Remark 1 .

Observe that Proposition 2 follows directly from Proposition 3. 
Proof of Proposition 3. Let $\pi_{1}(X)=\sqrt{n} /(1-\varepsilon)$. By Step 1, (9), and (19) we get

$$
2 \alpha / \sqrt{n}+(\alpha / \sqrt{n}-1)^{2} \geq f_{0}^{n} .
$$

Let $\alpha=\sqrt{n+2}$ if $\mathbf{K}=\mathbf{R}$ and $\alpha=\sqrt{n+1}$ if $\mathbf{K}=\mathbf{C}$. A calculation using formula (18) for $f_{0}^{n}$ implies a lower bound for $\varepsilon$ which turns out to yield

$$
\pi_{1}\left(X^{\mathbf{R}}\right) \geq n / f(n, n(n+1) / 2), \quad \pi_{1}\left(X^{\mathbf{C}}\right) \geq n / f\left(n, n^{2}\right),
$$

where $f(n, N)$ is the function given in (3).

Proof of Remark 3. We indicate the necessary changes in Step 1 of the previous argument to get a lower bound for $\pi_{p}(X)$ if $1<p<2$. Taking a $p$-summing factorization of $\mathrm{Id}_{X}$, one finds again a compact space $T$ and a probability measure $\mu$ on $T$ and the isometric image $X_{\infty} \subset L_{\infty}(T, \mu)$ of $X$ such that

$$
\|g\|_{\infty} \leq \pi_{p}(X)\left(\int_{T}|g(s)|^{p} d \mu(s)\right)^{1 / p} .
$$

In $X_{p} \subset L_{p}(T, \mu)$ there is a basis $\left(f_{j}\right)_{j=1}^{n}$ such that

$$
n \int_{T} f_{j}(s) \overline{f_{k}(s)} f(s)^{p-2} d \mu(s)=\delta_{j k},
$$

where $f=\left(\sum_{j=1}^{n}\left|f_{j}\right|^{2}\right)^{1 / 2} \quad$ [Lew1]. Let $d \nu(s)=f(s)^{p} d \mu(s), \quad h_{j}(s):=$ $\sqrt{n} f_{j}(s) / f(s)$, and $h(s, t):=\sum_{j=1}^{n} h_{j}(s) \overline{h_{j}(t)}$. Then the orthogonality relations (7) hold and the previous arguments show that

$$
\begin{aligned}
n & \leq \pi_{p}(X) \int_{T}\left(\int_{T}\left|\sum_{j=1}^{n} n f_{j}(s) \overline{f_{j}(t)}\right|^{p} d \mu(t)\right)^{1 / p} d \mu(s) \\
& \leq \pi_{p}(X)\left(\int_{T} \int_{T}|h(s, t)|^{p} d \nu(t) d \nu(s)\right)^{1 / p}=\pi_{p}(X)\|h\|_{p} .
\end{aligned}
$$

By (7), $\|h\|_{2}=\sqrt{n}$; hence Hölder's inequality shows that

$$
\|h\|_{p} \leq\|h\|_{1}^{2 / p-1}\|h\|_{2}^{2 / p^{\prime}}=n^{1 / p^{\prime}}\|h\|_{1}^{2 / p-1} .
$$

Define $\varepsilon \geq 0$ by $\pi_{p}(X)=\sqrt{n} /(1-\varepsilon)$. We get

$$
\int_{T} \int_{T}|h(s, t)| d v(s) d v(t) \geq\left(\frac{n^{1 / p}}{\pi_{p}(X)}\right)^{p /(2-p)}=(1-\varepsilon)^{p /(2-p)} \sqrt{n} .
$$

As in (9), this yields (with $\alpha=\sqrt{n}$ )

$$
\begin{aligned}
I & =\int_{T} \int_{T}\left(\frac{1}{\sqrt{n}}|h(s, t)|-1\right)^{2} d \nu(s) d \nu(t) \\
& \leq 2\left(1-(1-\varepsilon)^{p /(2-p)}\right) \leq\left(1 / p-\frac{1}{2}\right)^{-1} \varepsilon .
\end{aligned}
$$


Since $I \geq f_{0}^{n}$ and $f_{0}^{n}=2 / n-O\left(1 / n^{3 / 2}\right)$ if $\mathbf{K}=\mathbf{R}$ and $f_{0}^{n}=1 / n-O\left(1 / n^{3 / 2}\right)$ if $\mathbf{K}=\mathbf{C}$, we conclude

$$
\begin{array}{ll}
\pi_{p}(X) \geq \sqrt{n}+(2 / p-1) / \sqrt{n}-O(1 / n) & \text { if } \mathbf{K}=\mathbf{R}, \\
\pi_{p}(X) \geq \sqrt{n}+\left(1 / p-\frac{1}{2}\right) / \sqrt{n}-O(1 / n) & \text { if } \mathbf{K}=\mathbf{C} .
\end{array}
$$

\section{SPACES With NEARLy EXTREMAL $\lambda-, \pi_{1}-$, AND gl-CONSTANTS}

We now construct a class of spaces with very large projection constants and very small 1-summing constants. The spaces are of a concrete nature: they are translation-invariant subspaces of $l_{\infty}^{N}$, the elements of $l_{\infty}^{N}$ being considered as functions on a finite group. They may also be constructed to have GordonLewis constants of maximal order and extremal Euclidean distance and Sidon constants, while being character spaces over $\Lambda_{4}$-sets. The estimates for $\lambda$ and $\pi_{1}$ are based on the main idea of [Kö], which can be formulated in the following lemma. In the formulae below, $\|\cdot\|_{2}$ denotes the usual $l_{2}$-norm on $\mathbf{K}^{n}$ and $\mathbf{K}^{N}$.

Lemma 6. Let $A$ be a scalar $N \times n$ matrix of rank $n$ and $N>n$. We denote the $N$ rows of $A$ by $x_{s} \in \mathbf{K}^{n}$ and the $n$ columns of $A$ by $y_{j} \in \mathbf{K}^{N}$ and let $X_{\infty}^{n}:=\operatorname{Span}\left[y_{1}, \ldots, y_{n}\right] \subseteq l_{\infty}^{N}$, equipped with the $l_{\infty}^{N}$-norm. If for some $0<\alpha<1$

$$
\left\|x_{s}\right\|_{2}=\sqrt{n} \text { and }\left|\left\langle x_{s}, x_{t}\right\rangle\right| \leq \alpha n \text { for all } s \neq t, 1 \leq s, t \leq N,
$$

then $\operatorname{dim} X_{\infty}^{n}=n$ and

$$
\lambda\left(X_{\infty}^{n}\right) \geq(1-n / N)(1 / \alpha)+n / N .
$$

If additionally

$$
\left\|y_{j}\right\|_{2}=\sqrt{N} \text { and }\left\langle y_{j}, y_{k}\right\rangle=0 \text { for all } j \neq k, 1 \leq j, k \leq n,
$$

then

$$
\pi_{1}\left(X_{\infty}^{n}\right) \leq N n \alpha /(N-n+\alpha n) .
$$

If $\alpha$ satisfies $\alpha=\sqrt{(N-n) / n(N-1)}$, equality holds in (21) and (23) and $\lambda\left(X_{\infty}^{n}\right) \pi_{1}\left(X_{\infty}^{n}\right)=n$.

Remark 5. The vectors $x_{s}$ form equiangular lines if for all $s \neq t,\left|\left\langle x_{s}, x_{t}\right\rangle\right|=$ $\alpha n$ holds. For $\alpha=\sqrt{(N-n) /(n(N-1))}$ the inequality in (20) actually implies the equality $\left|\left\langle x_{s}, x_{t}\right\rangle\right|=\alpha n$.

Clearly, the norm of a vector $\sum_{j=1}^{n} \lambda_{j} y_{j} \in X_{\infty}^{n}$ is just given by

$$
\left\|\sum_{j=1}^{n} \lambda_{j} y_{j}\right\|_{\infty}=\sup _{1 \leq s \leq N}\left|\left\langle\lambda, x_{s}\right\rangle\right|,
$$


where $\lambda=\left(\lambda_{j}\right)_{j=1}^{n}$ and $\langle\cdot, \cdot\rangle$ denotes the scalar product in $\mathbf{K}^{n}$. An application of the Hahn-Banach theorem shows that the dual space $\left(X_{\infty}^{n}\right)^{*}$ has the absolutely convex hull of the vectors $\left(x_{s}\right)_{s=1}^{N} \subset \mathbf{K}^{n}$ as its unit ball. Clearly, (22) implies that $A$ has rank $n$, but (20) by itself does not necessarily imply it.

Proof. Let $e_{j} \in \mathbf{K}^{n}$ denote the standard unit vector basis. Since $y_{j}=A e_{j}$, $A A^{*}=\left(\left\langle x_{s}, x_{t}\right\rangle\right)_{s, t=1}^{N}$ maps $l_{\infty}^{N}$ into $X_{\infty}^{n}$. Let $\operatorname{Id}_{N}$ denote the $N \times N$ identity matrix. Then

$$
B=\left(b_{s t}\right)_{s, t=1}^{N}:=\frac{1}{(1-\alpha) n}\left(\left\langle x_{s}, x_{t}\right\rangle\right)_{s, t=1}^{N}-\operatorname{Id}_{N}: l_{\infty}^{N} \rightarrow l_{\infty}^{N}
$$

maps $X_{\infty}^{N}$ into itself. One has

$$
\operatorname{tr}\left(\left.B\right|_{X_{\infty}^{n}}\right)=\frac{N}{1-\alpha}-n, \quad\left|b_{s t}\right| \leq \frac{\alpha}{1-\alpha} \quad \text { for all } 1 \leq s, t \leq N .
$$

The trace is always bounded by the nuclear norm (cf., e.g., [TJ]) and thus

$$
\left|\operatorname{tr}\left(\left.B\right|_{X_{\infty}^{n}}\right)\right| \leq \nu\left(\left.B\right|_{X_{\infty}^{n}}\right) \leq \lambda\left(X_{\infty}^{n}\right) \nu\left(B: l_{\infty}^{N} \rightarrow l_{\infty}^{N}\right) .
$$

On $l_{\infty}^{N}$, the nuclear norm is $\nu(B)=\sum_{t=1}^{n} \sup _{1 \leq s \leq N}\left|b_{s t}\right| \leq N \alpha /(1-\alpha)$ (cf., e.g., $[\mathrm{TJ}])$. Hence

$$
\lambda\left(X_{\infty}^{n}\right) \geq \frac{1}{\alpha}-\frac{n}{N} \frac{1-\alpha}{\alpha}=\left(1-\frac{n}{N}\right) \frac{1}{\alpha}+\frac{n}{N} .
$$

If additionally (22) is satisfied, i.e., $A^{*} A=N \operatorname{Id}_{n}$, then $P:=(1 / N) A A^{*}$ is a projection onto $X_{\infty}^{N}$. Indeed, $\operatorname{tr}(P)=n$ and

$$
P y_{j}=(1 / N)\left(A A^{*}\right) A e_{j}=A e_{j}=y_{j} \quad \text { for } 1 \leq j \leq n \text {. }
$$

Therefore

$$
\lambda\left(X_{\infty}^{n}\right) \leq\|P\|=\sup _{1 \leq s \leq N} \sum_{t=1}^{N} \frac{1}{N}\left|\left\langle x_{s}, x_{t}\right\rangle\right|=\frac{n}{N}(1+(N-1) \alpha) .
$$

For $\alpha=\sqrt{(N-n) / n(N-1)}$ this is equal to $(1-n / N)(1 / \alpha)+n / N$ and equality holds in (21). Assuming (22), $B$ is a multiple of the Id on $X_{\infty}^{n}$,

$$
\left.B\right|_{X_{\infty}^{n}}=\left(\frac{N}{n} \frac{1}{1-\alpha}-1\right) \operatorname{Id}_{X_{\infty}^{n}} .
$$

Since the 1-summing norm is injective and bounded by the nuclear norm, we find

$$
\begin{gathered}
\left(\frac{N}{n} \frac{1}{1-\alpha}-1\right) \pi_{1}\left(X_{\infty}^{n}\right)=\pi_{1}\left(\left.B\right|_{X_{\infty}^{n}}\right) \leq \nu(B)=\frac{N \alpha}{1-\alpha}, \\
\pi_{1}\left(X_{\infty}^{n}\right) \leq N n \alpha /(N-n(1-\alpha)) .
\end{gathered}
$$

This shows (23). For $\alpha=\sqrt{(N-n) / n(N-1)}$, the trace-duality (1) and the formula for $\lambda\left(X_{\infty}^{n}\right)$ yield the equality in (23) since

$$
n \leq \lambda\left(X_{\infty}^{n}\right) \pi_{1}\left(X_{\infty}^{n}\right) \leq \frac{N-n+n \alpha}{N \alpha} \cdot \frac{N n \alpha}{N-n(1-\alpha)}=n,
$$

completing the proof. 
Proof of Remark 2. The upper estimate follows from Proposition 2. If $n=3$ the six diagonals of the dodecahedron in $\mathbf{R}^{3}$ yield six equiangular (normalized in $l_{2}^{3}$ ) vectors $x_{s} \in \mathbf{R}^{3}$ with $\left|\left\langle x_{s}, x_{t}\right\rangle\right|=1 / \sqrt{5}$, for $s \neq t, 1 \leq s, t \leq 6$. By (21), the corresponding space $X_{\infty}^{3}$ satisfies $\lambda\left(X_{\infty}^{3}\right) \geq(1+\sqrt{5}) / 2$. The dual unit ball of $X_{\infty}^{3}$ is the icosahedron (= absolutely convex hull of the $x_{s}$ 's); hence the unit ball of $X_{\infty}^{3}$ is the dodecahedron. For $n=2$ take the three diagonals of the (self-dual) regular hexagon in $\mathbf{R}^{2}$. Condition (22) is satisfied in both cases and $\alpha=\sqrt{(N-n) / n(N-1)}$ holds, too.

Proof of Proposition 4(b). The Kerdock code [Ke] (see also Levenštein [Lev]) shows that for $n=4^{m}$ there exist $N=n^{2} / 2$ real vectors $x_{s} \in \mathbf{R}^{n}$ with coordinates \pm 1 and $\left\|x_{s}\right\|_{2}=\sqrt{n},\left|\left\langle x_{s}, x_{t}\right\rangle\right| \leq \sqrt{n}$ for $s \neq t, 1 \leq s, t \leq N$. Lemma 6 shows that the corresponding space $X_{\infty}^{n}$ satisfies

$$
\lambda\left(X_{\infty}^{n}\right) \geq\left(1-\frac{n}{N}\right) \sqrt{n}+\frac{n}{N}=\sqrt{n}-\frac{2}{\sqrt{n}}+\frac{2}{n} .
$$

On the other hand, by formula (3),

$$
\lambda\left(X_{\infty}^{n}\right) \leq f\left(n, n^{2} / 2\right) \leq \sqrt{n}-1 / \sqrt{n} .
$$

We proceed now to the main construction of $n$-dimensional translationinvariant subspaces of $l_{\infty}^{N}$ which have extremal properties stated in Propositions 4 and 5 . Such a subspace will be defined by a sequence of nonnegative integers $d_{1}, \ldots, d_{n}$, which are distinct $\bmod N$. Set

$$
y_{j}:=\left(\exp \left(\frac{2 \pi i}{N} d_{j} s\right)\right)_{s=0}^{N-1} \in \mathbf{C}^{n} \text { for } j=1, \ldots, n .
$$

Then put $X_{\infty}^{n}:=\operatorname{Span}\left[y_{1}, \ldots, y_{n}\right] \subset l_{\infty}^{N}$, equipped with the $l_{\infty}$-norm. Clearly, $\operatorname{dim} X_{\infty}^{n}=n$. The $y_{j}$ 's can be identified with characters on the cyclic group $\mathbf{Z}_{N}$. In fact, for $j=1, \ldots, n$ and $s \in\{0, \ldots, N-1\}=\mathbf{Z}_{N}$,

$$
y_{j}(s)=\exp \left((2 \pi i / N) d_{j} s\right) .
$$

In particular, $X_{\infty}^{n}$ is a translation-invariant space.

The properties of $X_{\infty}^{n}$ depend on number theoretic properties of the defining sequence $d_{1}, \ldots, d_{n}$. In particular, we shall use some facts about so-called finite $B_{2}$-sequences, which can be found, e.g., in [HR].

Theorem 7. Let $m \in \mathbf{N}, p$ be a prime number, and $q=p^{m}$.

(a) Let $M=q^{2}-1$. Then there exist $q$ integers $0 \leq d_{1}, \ldots, d_{q}<M$ such that the $q(q-1)=M-(q-1)$ differences $d_{i}-d_{j}, i \neq j$, when reduced $\bmod M$, are all the positive integers less than $M$ which are not divisible by $(q+1)$.

(b) Let $N=q^{2}+q+1$. Then there exist $(q+1)$ integers $0 \leq d_{0}, \ldots, d_{q}<N$ such that the $q(q+1)=N-1$ differences $d_{i}-d_{j}, i \neq j$, when reduced $\bmod N$, are all the positive integers less than $N$. 
Now, let $m \in \mathbf{N}, p$ be a prime number, $q=p^{m}$. Let $n:=q+1$ and $N:=q^{2}+q+1=n^{2}-n+1$. Let $d_{0}, d_{1}, \ldots, d_{q}$ be the sequence from Theorem $7(\mathrm{~b})$ and let $y_{j}$ be defined by (24), for $j=0, \ldots, q$. The space $X_{\infty}^{n}$ satisfies the requirement of Proposition 4(a), i.e.,

$$
\lambda\left(X_{\infty}^{n}\right)=f(n, N) \text { and } \pi_{1}\left(X_{\infty}^{n}\right)=n / f(n, N) .
$$

Proof of Proposition 4(a). Let $\alpha=\sqrt{(N-n) / n(N-1)}=\sqrt{(n-1) / n}$. Then $(1-n / N)(1 / \alpha)+n / N=f(n, N)$. By Lemma 6, it suffices to check (20) and (22) for the matrix $A$ built with column vectors $y_{j}$. Clearly $\left\|y_{j}\right\|_{2}=\sqrt{N}$ and

$$
\left\langle y_{j}, y_{k}\right\rangle=\frac{1}{N} \sum_{s=0}^{N-1} \exp \left(\frac{2 \pi i}{N}\left(d_{j}-d_{k}\right) s\right)=0 \text { for } j \neq k, 0 \leq j, k \leq q .
$$

For the row vectors $x_{s}$ we have $\left\|x_{s}\right\|_{2}=\sqrt{n}$, for $s=0, \ldots, N-1$. Moreover, for $s \neq t, 0 \leq s, t \leq N-1$ we have, with $\theta=s-t$,

$$
\begin{aligned}
\left|\left\langle x_{s}, x_{t}\right\rangle\right|^{2} & =\left|\sum_{j=0}^{q} \exp \left(\frac{2 \pi i}{N} d_{j} \theta\right)\right|^{2}=\sum_{j, k=0}^{q} \exp \left(\frac{2 \pi i}{N}\left(d_{j}-d_{k}\right) \theta\right) \\
& =\left\{\sum_{j=k} \exp \left(\frac{2 \pi i}{N}\left(d_{j}-d_{k}\right) \theta\right)+\sum_{j \neq k} \exp \left(\frac{2 \pi i}{N}\left(d_{j}-d_{k}\right) \theta\right)\right\} \\
& =n+\sum_{l=1}^{N-1} \exp \left(\frac{2 n i}{N} l \theta\right)=n-1,
\end{aligned}
$$

since $\sum_{l=0}^{N-1} \exp ((2 \pi i / N) l \theta)=0$. Hence $\left|\left\langle x_{s}, x_{t}\right\rangle\right|=\alpha n$ for $s \neq t$, completing the proof.

Note that since $X_{\infty}^{n}$ is translation invariant a general averaging argument shows that $\lambda\left(X_{\infty}^{n}\right)$ is equal to the norm of the orthogonal projection from $l_{\infty}^{N}$ onto $X_{\infty}^{n}$. Since the projection $P$ constructed in the proof of Lemma 6 is orthogonal, for translation-invariant spaces the first part of the proof of Lemma 6 can be omitted.

The proof above shows that the $x_{s}$ 's form equiangular vectors in $\mathbf{C}^{n}$. In fact, by Levenštein [Lev], for this $\alpha=\sqrt{n-1} / n$, the number $N$ is maximal. This example also shows that the lower estimate of the double integral in Step 4 of the previous section and in the proof of Proposition 3 is almost the best possible, in general (up to terms of $O\left(n^{3 / 2}\right)$ ).

Proposition 4 is a slight improvement of the result from [Kö], where a similar construction was considered, with $N=q^{2}$ and $d_{j}=j^{2}, j=1, \ldots, q=n$. This gave $X_{\infty}^{n}$ such that $\lambda\left(X_{\infty}^{n}\right)=\sqrt{n}-1 / \sqrt{n}+O(1 / n)$, while here we have $\lambda\left(X_{\infty}^{n}\right)=\sqrt{n}-1 / 2 \sqrt{n}+O(1 / n)$.

We pass now to the main construction of this section, which was announced in Proposition 5. Let $m \in \mathbf{N}$ and $p$ be an odd prime number. Set $n:=p^{m}$, $M:=n^{2}-1$, and $N:=M / 2=(n-1)(n+1) / 2$. Let $0 \leq d_{1}, \ldots, d_{n}<M$ be 
the sequence from Theorem 7(a) such that the differences $d_{i}-d_{j}$, for $i \neq j$, $1 \leq i, j \leq n$, when reduced $\bmod M$, are all the positive integers less than $M$ which are not divisible by $(n+1)$.

Let $y_{j} \in \mathbf{C}^{N}(j=1, \ldots, n)$ be defined by $(24)$, i.e.,

$$
y_{j}:=\left(\exp \left((2 \pi i / N) d_{j} s\right)\right)_{s=0}^{N-1} \in \mathbf{C}^{N} \text { for } j=1, \ldots, n .
$$

Let $X_{\infty}^{n}:=\operatorname{Span}\left[y_{1}, \ldots, y_{n}\right] \subset l_{\infty}^{N}$.

Let $\|\cdot\|_{p}$ denote the $L_{p}$-form on $\mathbf{C}^{N}$ in the function normalization, for $1 \leq$ $p<\infty$, i.e., for $y=(y(s))_{s=0}^{N-1} \in \mathbf{C}^{N},\|y\|_{p}=\left((1 / N) \sum_{s=0}^{N-1}|y(s)|^{p}\right)^{1 / p}$. Hence $\|y\|_{p} \leq\|y\|_{q}$ for $p \leq q$. Let $L_{p}^{N}=\left(\mathbf{C}^{N},\|\cdot\|_{p}\right)$ and $X_{p}^{n}:=\operatorname{Span}\left[y_{1}, \ldots, y_{n}\right] \subset$ $L_{p}^{N}$, with the inherited $L_{p}$-norm.

Now we are ready to show that the space $X_{\infty}^{n}$ satisfies the conclusions of Proposition 5.

Proof of Proposition 5. (a), (b) Let $\alpha=1 / \sqrt{n}$. Let $x_{s} \in \mathbf{C}^{n} \quad(s=0, \ldots, N-$ 1) be the row vectors of the $N \times n$ matrix $A$ built from column vectors $y_{1}, \ldots, y_{n}$. We check that (20) and (22) of Lemma 6 hold. Clearly, $\|y\|_{2}=\sqrt{N}$ and for $j \neq k, 1 \leq j, k \leq n$,

$$
\left\langle y_{j}, y_{k}\right\rangle=\sum_{s=0}^{N-1} \exp \left(\frac{2 \pi i}{N}\left(d_{j}-d_{k}\right) s\right)=0 .
$$

The vectors $\left(x_{s}\right)_{s=0}^{N-1} \in l_{2}^{n}$ are almost equiangular; for $s \neq t, 0 \leq s, t \leq N-1$ we have with $\theta:=s-t$,

$$
\begin{aligned}
\left|\left\langle x_{s}, x_{t}\right\rangle\right|^{2} & =\left|\sum_{j=1}^{n} \exp \left(\frac{2 \pi i}{N} d_{j} \theta\right)\right|^{2}=\sum_{j, k=1}^{n} \exp \left(\frac{2 \pi i}{N}\left(d_{j}-d_{k}\right) \theta\right) \\
& =\left\{\sum_{j=k} \exp \left(\frac{2 \pi i}{N}\left(d_{j}-d_{k}\right) \theta\right)+\sum_{j \neq k} \exp \left(\frac{2 \pi i}{N}\left(d_{j}-d_{k}\right) \theta\right)\right\} \\
& =n+\sum_{l=1}^{M-1} \exp \left(\frac{2 \pi i}{N} l \theta\right)-\sum_{m=1}^{n-2} \exp \left(\frac{2 \pi i}{N} m(n+1) \theta\right) \\
& =n-1-\sum_{m=1}^{n-2} \exp \left(\frac{2 \pi i}{n-1} 2 \theta m\right) .
\end{aligned}
$$

If $\theta=s-t$ is not divisible by $(n-1)$, the last sum is equal to -1 ; otherwise it is $(n-2)$. Therefore

$$
\left|\left\langle x_{s}, x_{t}\right\rangle\right|= \begin{cases}\sqrt{n}, & (s-t)(\bmod M) \neq j(n-1), j=1, \ldots, n, \\ 1, & (s-t)(\bmod M)=j(n-1), j=1, \ldots, n .\end{cases}
$$


Thus Lemma 6 yields

$$
\begin{aligned}
& \lambda\left(X_{\infty}^{n}\right) \geq\left(1-\frac{n}{N}\right) \frac{1}{\alpha}+\frac{n}{N}=\sqrt{n}-\frac{2}{\sqrt{n}}+O\left(\frac{1}{n}\right), \\
& \pi_{1}\left(X_{\infty}^{n}\right) \leq \frac{N n \alpha}{N-n+n \alpha}=\sqrt{n}+\frac{2}{\sqrt{n}}-O\left(\frac{1}{n}\right) .
\end{aligned}
$$

Since $P:=(1 / N)\left(\left\langle x_{s}, x_{t}\right\rangle\right)_{s, t=0}^{N-1}: l_{\infty}^{N} \rightarrow l_{\infty}^{N}$ is a projection onto $X_{\infty}^{n}$, using (26) we find that

$$
\begin{aligned}
\lambda\left(X_{\infty}^{n}\right) & \leq\|P\|=\sup _{0 \leq s \leq N-1} \sum_{t=0}^{N-1} \frac{1}{N}\left|\left\langle x_{s}, x_{t}\right\rangle\right| \\
& =\frac{n}{N}\left(1+(N-(n+1)) \frac{1}{\sqrt{n}}+n \cdot \frac{1}{n}\right)=\sqrt{n}-\frac{2}{\sqrt{n}}+O\left(\frac{1}{n}\right) .
\end{aligned}
$$

By trace-duality,

$$
\pi_{1}\left(X_{\infty}^{n}\right) \geq n / \lambda\left(X_{\infty}^{n}\right)=\sqrt{n}+2 / \sqrt{n}-O(1 / n),
$$

proving (a) and (b).

(c) For an arbitrary $n$-dimensional space $X_{n}, d\left(X_{n}, l_{2}^{n}\right) \leq \sqrt{n}$, and this estimate is given by the so-called John's ellipsoid of maximal volume, contained in the unit ball $B_{E}$ (cf., e.g., [TJ, $\left.\S \S 9,15\right]$ ). Therefore we need to show that $d\left(X_{\infty}^{n}, l_{2}^{n}\right) \geq \sqrt{n}$. Clearly $X_{2}^{n}$ and $l_{2}^{n}$ are isometric. Let $\beta_{j}:=\exp \left((2 \pi i / N) d_{j}\right)$ for $j=1, \ldots, n$. Then $I: X_{\infty}^{n} \rightarrow X_{\infty}^{n}$ defined by $\sum_{j=1}^{n} a_{j} y_{j} \rightarrow \sum_{j=1}^{n} \beta_{j} a_{j} y_{j}$ is an isometry and $I^{N}=\operatorname{Id}_{X_{\infty}^{n}}$. Any inner product $[\cdot, \cdot]$ on $X_{\infty}^{n}$ invariant with respect to $I$ is diagonal in the basis $\left(y_{j}\right)$. Indeed, if $[x, z]=\sum_{j, k=1}^{n} t_{j k} a_{j} \overline{b_{k}}$, for $x=\sum_{j=1}^{n} a_{j} y_{j}$ and $z=\sum_{k=1}^{n} b_{k} y_{k}$, then the condition $[I x, I z]=[x, z]$ implies that $t_{j k}=t_{j k} \beta_{j} \bar{\beta}_{k}$. For $j \neq k$, however,

$$
\beta_{j} \bar{\beta}_{k}=\exp \left((2 \pi i / N)\left(d_{j}-d_{k}\right)\right) \neq 1
$$

and thus $t_{j k}=0$. Note here that $d_{j}-d_{k} \neq N(\bmod N)$ since $N$ is divisible by $(n+1)$. Now let $(\cdot, \cdot)$ be an inner product on $X_{\infty}^{n}$ which determines the distance $d\left(X_{\infty}^{n}, l_{2}^{n}\right)=d$, normalized so that

$$
\left(1 / d^{2}\right)\|x\|_{\infty}^{2} \leq(x, x) \leq\|x\|_{\infty}^{2} \text { for } x \in X_{\infty}^{n} .
$$

Define the inner product $[\cdot, \cdot]$ on $X_{\infty}^{n}$ by

$$
[x, z]=\frac{1}{N} \sum_{k=0}^{N-1}\left(I^{k} x, I^{k} z\right) \quad \text { for } x, z \in X_{\infty}^{n} .
$$

Clearly,

$$
\left(1 / d^{2}\right)\|x\|_{\infty}^{2} \leq[x, x] \leq\|x\|_{\infty}^{2} \text { for } x \in X_{\infty}^{n} .
$$

Moreover, $[x, z]=[I x, I z]$ for $x, z \in X_{\infty}^{n}$. Therefore there exist positive numbers $\lambda_{1}, \ldots, \lambda_{n}$ such that

$$
[x, z]=\sum_{j=1}^{n} \lambda_{j} a_{j} \bar{b}_{j}
$$


for $x=\sum_{j=1}^{n} a_{j} y_{j}$ and $z=\sum_{j=1}^{n} b_{j} y_{j}$. Since $[x, x] \leq\|x\|_{\infty}^{2}$, then $\lambda_{j} \leq 1$ for $j=1, \ldots, n$.

Set $z:=\sum_{j=1}^{n} y_{j}$. Then $[z, z]=\sum_{j=1}^{n} \lambda_{j}$. Taking $s=0$, we find

$$
\|z\|_{\infty}=\left\|\sum_{j=1}^{n} y_{j}\right\|_{\infty}=\sup _{0 \leq s \leq N-1}\left|\sum_{j=1}^{n} \exp \left(\frac{2 \pi i}{N} d_{j} s\right)\right|=n \text {. }
$$

So

$$
d \geq \sup _{x \neq 0}\|x\|_{\infty} /[x, x]^{1 / 2} \geq \frac{n}{\left(\sum_{j=1}^{n} \lambda_{j}\right)^{1 / 2}} \geq \sqrt{n},
$$

since $\lambda_{j} \leq 1$ for $j=1, \ldots, n$. Thus $d\left(X_{\infty}^{n}, l_{2}^{n}\right)=d\left(X_{\infty}^{n}, X_{2}^{n}\right)=d=\sqrt{n}$. Also, $\lambda_{j}=1$ for $j=1, \ldots, n$; hence the distance $d\left(X_{\infty}^{n}, l_{2}^{n}\right)$ is given by the inclusion map $I_{\infty 2}: X_{\infty}^{n} \rightarrow X_{2}^{n}$. In fact, the natural inner product is the only inner product diagonal with respect to the character basis $\left(y_{j}\right)$, which determines the distance.

(d) Set $z:=\sum_{j=1}^{n} y_{j}$. By (28), $\|z\|_{\infty}=n$. Set $\varepsilon_{j}:=\exp \left((2 \pi i / M) d_{j}\right)$, for $j=1, \ldots, n$ and $w:=\sum_{j=1}^{n} \varepsilon_{j} y_{j}$. Then

$$
\begin{aligned}
\|w\|_{\infty}^{2} & =\sup _{0 \leq s \leq N-1}\left|\sum_{j=1}^{n} \exp \left(\frac{2 \pi i}{M} d_{j}(2 s+1)\right)\right|^{2} \\
& =\sup _{0 \leq s \leq N-1} \sum_{j, k=1}^{n} \exp \left(\frac{2 \pi i}{M}\left(d_{j}-d_{k}\right)(2 s+1)\right) \\
& =\sup _{0 \leq s \leq N-1}\left[n+\sum_{j \neq k} \exp \left(\frac{2 \pi i}{M}\left(d_{j}-d_{k}\right)(2 s+1)\right)\right] \\
& =n .
\end{aligned}
$$

For the last equality observe that since $(2 \pi i / M)(n+1)=2 \pi i /(n-1)$, the latter sum, for every $0 \leq s \leq N-1$, is equal to

$$
\sum_{l=1}^{m-1} \exp \left(\frac{2 \pi i}{M} l(2 s+1)\right)-\sum_{m=1}^{n-2} \exp \left(\frac{2 \pi i}{M} m(n+1)(2 s+1)\right)=-1-(-1)=0 \text {. }
$$

Thus $\|w\|_{\infty}=\sqrt{n}$. Therefore $\operatorname{ubc}\left(y_{j}\right) \geq\|z\|_{\infty} /\|w\|_{\infty}=\sqrt{n}$. On the other hand, an arbitrary character basis $\left(y_{j}\right)$ in a translation-invariant space satisfies $\operatorname{ubc}\left(y_{j}\right) \leq \sqrt{n}$. Indeed, observe that the uniqueness of the John's ellipsoid of maximal volume (cf. [TJ, §15]) implies that the translation operator $I$, defined in (c), is an isometry in the Hilbert space $H$ associated to this ellipsoid. This yields that $\left(y_{j}\right)$ form an orthogonal, hence also 1-unconditional, basis in $H$. So $\operatorname{ubc}\left(y_{j} \mid X_{\infty}^{n}\right) \leq \sqrt{n} \operatorname{ubc}\left(y_{j} \mid H\right)=\sqrt{n}$.

(e) The vectors $\left(y_{j}\right)_{j=1}^{n}$ form the natural character basis in the translationinvariant space $X_{\infty}^{n}$ (cf. [25]) and so the Sidon constant $S\left(X_{\infty}^{n}\right)$ is the smallest 
constant $c>0$ such that, for all $\left(a_{j}\right)_{j=1}^{n} \in \mathbf{C}^{n}$,

$$
\sum_{j=1}^{n}\left|a_{j}\right| \leq c\left\|\sum_{j=1}^{n} a_{j} y_{j}\right\|_{\infty} .
$$

Since the left-hand side norm is 1-unconditional, it easily follows by the triangle inequality that $\operatorname{ubc}\left(y_{j}\right) \leq S\left(X_{\infty}^{n}\right)$. On the other hand, we have

$$
\begin{aligned}
\sum_{j=1}^{n}\left|a_{j}\right| & =\sup _{0 \leq s \leq N-1} \sup _{\left|\eta_{j}\right|=1}\left|\sum_{j=1}^{n} \eta_{j} a_{j} \exp \left(\frac{2 \pi i}{N} d_{j} s\right)\right| \\
& =\sup _{\left|\eta_{j}\right|=1}\left\|\sum_{j=1}^{n} \eta_{j} a_{j} y_{j}\right\|_{\infty} \leq \mathrm{ubc}\left(y_{j}\right)\left\|\sum_{j=1}^{n} a_{j} y_{j}\right\|_{\infty},
\end{aligned}
$$

and so $S\left(X_{\infty}^{n}\right) \leq \operatorname{ubc}\left(y_{j}\right)$. Thus, by (d), $S\left(X_{\infty}^{n}\right)=\sqrt{n}$ and this is the maximal value for any Sidon constant.

(f) First observe that on $X_{\infty}^{n}$ the $L_{2}$ - and $L_{4}$-norms are equivalent. Precisely,

$$
\|y\|_{2} \leq\|y\|_{4} \leq\left(3^{1 / 4}\right)\|y\|_{2} \text { for } y \in X_{\infty}^{n} .
$$

Inequality (29) is well known in harmonic analysis, in much more general form, and it says that the $y_{j}$ 's form a $\Lambda_{4}$-set (cf., e.g., [R]). Its proof consists of a straightforward calculation evaluating the fourth power $\|y\|_{4}^{4}$ and depends on the fact that every integer $0<l \leq N-1$ can be represented as a difference $l=d_{j}-d_{k}$ in at most one way. As a standard consequence of (29), we get by Hölder's inequality

$$
\|y\|_{2} \leq\|y\|_{4}^{2 / 3}\|y\|_{1}^{1 / 3} \leq 3^{1 / 6}\|y\|_{2}^{2 / 3}\|y\|_{1}^{1 / 3},
$$

so that

$$
\|y\|_{2} \leq \sqrt{3}\|y\|_{1} \text { for } y \in X_{\infty}^{n} .
$$

Now an argument from [Pis] shows that

$$
\operatorname{ubc}\left(y_{j}\right)=\operatorname{ubc}\left(y_{j} \mid X_{\infty}^{n}\right) \leq 3 \operatorname{gl}\left(X_{\infty}^{n}\right) .
$$

Let us briefly describe how the argument works in our situation.

As in (c), consider the isometry $I: X_{\infty}^{n} \rightarrow X_{\infty}^{n}$ defined by

$$
I\left(\sum_{j=1}^{n} a_{j} y_{j}\right)=\sum_{j=1}^{n} \exp \left(\frac{2 \pi i}{N} d_{j}\right) a_{j} y_{j} \text { for } \sum_{j=1}^{n} a_{j} y_{j} \in X_{\infty}^{n} .
$$

In fact, $I$ is the restriction to $X_{\infty}^{n}$ of the translation operator $\tilde{I}: \mathbf{C}^{N} \rightarrow \mathbf{C}^{N}$ defined by $\tilde{I}\left(\alpha_{0}, \ldots, \alpha_{N-1}\right)=\left(\alpha_{1}, \ldots, \alpha_{N-1}, \alpha_{0}\right)$. For $0 \leq s \leq N-1$ put

$$
w^{s}=I^{s} w \quad \text { for } w \in X_{\infty}^{n} ; \quad\left(w^{*}\right)^{s}=\left(I^{*}\right)^{s} w^{*} \quad \text { for } w^{*} \in\left(X_{\infty}^{n}\right)^{*} .
$$

Clearly, $\left\|w^{s}\right\|_{\infty}=\|w\|_{\infty}$ and $\left\|\left(w^{*}\right)^{s}\right\|_{\infty}^{*}=\left\|w^{*}\right\|_{\infty}^{*}$ (here $\|\cdot\|_{\infty}^{*}$ denotes the norm on $\left.\left(X_{\infty}^{n}\right)^{*}\right)$. 
Fix arbitrary $\left(\delta_{j}\right) \subset \mathbf{C}$ with $\left|\delta_{j}\right|=1$ and $\left(a_{j}\right),\left(b_{j}\right) \subset \mathbf{C}$ for $j=1, \ldots, n$. Let $w:=\sum_{j=1}^{n} a_{j} y_{j} \in X_{\infty}^{n}$ and define $w^{*} \in\left(X_{\infty}^{n}\right)^{*}$ by $\left\langle w^{*}, y_{j}\right\rangle=b_{j}$. Consider $A:\left(X_{\infty}^{n}\right)^{*} \rightarrow X_{2}^{n}$ and $B: X_{\infty}^{n} \rightarrow X_{2}^{n}$ given by

$$
\begin{gathered}
A x^{*}=\sum_{j=1}^{n}\left\langle x^{*}, y_{j}\right\rangle \delta_{j} a_{j} y_{j} \quad \text { for } x^{*} \in\left(X_{\infty}^{n}\right)^{*}, \\
B x=\sum_{j=1}^{n} b_{j} c_{j} y_{j} \quad \text { for } x=\sum_{j=1}^{n} c_{j} y_{j} \in X_{\infty}^{n} .
\end{gathered}
$$

We will estimate $\pi_{1}(A)$ and $\pi_{1}(B)$. By the orthogonality of the $y_{j}$ 's and (30),

$$
\begin{aligned}
\left\|A x^{*}\right\|_{2} & =\left(\sum_{j=1}^{n}\left|\left\langle x^{*}, y_{j}\right\rangle a_{j}\right|^{2}\right)^{1 / 2}=\left\|\sum_{j=1}^{n}\left\langle x^{*}, y_{j}\right\rangle a_{j} y_{j}\right\|_{2} \\
& \leq \sqrt{3}\left\|\sum_{j=1}^{n}\left\langle x^{*}, y_{j}\right\rangle a_{j} y_{j}\right\|_{1}=\sqrt{3} \frac{1}{N} \sum_{s=0}^{N-1}\left|\left\langle x^{*}, w^{s}\right\rangle\right| .
\end{aligned}
$$

Since $\left\|w^{s}\right\|_{\infty}=\|w\|_{\infty}$, this easily implies $\pi_{1}(A) \leq \sqrt{3}\|w\|_{\infty}$. For $B$ a similar calculation yields $\pi_{1}(B) \leq \sqrt{3}\left\|w^{*}\right\|_{\infty}^{*}$. Namely

$$
\begin{aligned}
\|B x\|_{2} & =\left(\sum_{j=1}^{n}\left|b_{j} c_{j}\right|^{2}\right)^{1 / 2}=\left\|\sum_{j=1}^{n} b_{j} c_{j} y_{j}\right\|_{2} \\
& \leq \sqrt{3}\left\|\sum_{j=1}^{n} b_{j} c_{j} y_{j}\right\|_{1}=\sqrt{3} \frac{1}{N} \sum_{s=0}^{N-1}\left|\left\langle\left(w^{*}\right)^{s}, x\right\rangle\right| .
\end{aligned}
$$

Since by trace-duality one has, with $\gamma_{1}(B)=\gamma_{\infty}\left(B^{*}\right)$,

$$
\begin{aligned}
\operatorname{gl}\left(X_{\infty}^{n}\right) & =\sup \left\{\gamma_{\infty}\left(B^{*}\right) / \pi_{1}(B) \mid 0 \neq B: X_{\infty}^{n} \rightarrow l_{2}^{n}\right\} \\
& =\sup \left\{\frac{\left|\operatorname{tr}\left(A B^{*}\right)\right|}{\pi_{1}(A) \pi_{1}(B)} \mid 0 \neq A:\left(X_{\infty}^{n}\right)^{*} \rightarrow l_{2}^{n}, 0 \neq B: X_{\infty}^{n} \rightarrow l_{2}^{n}\right\},
\end{aligned}
$$

then

$$
\begin{aligned}
\left|\left\langle w^{*}, \sum_{j=1}^{n} \delta_{j} a_{j} y_{j}\right\rangle\right| & =\left|\sum_{j=1}^{n} \delta_{j} a_{j} b_{j}\right|=\operatorname{tr}\left(A B^{*}\right) \\
& \leq \operatorname{gl}\left(X_{\infty}^{n}\right) \pi_{1}(A) \pi_{1}(B) \leq 3 \operatorname{gl}\left(X_{\infty}^{n}\right)\|w\|_{\infty}\left\|w^{*}\right\|_{\infty}^{*} .
\end{aligned}
$$

Passing to the supremum over $\left\|w^{*}\right\|_{\infty}^{*} \leq 1$ and then over $\left|\delta_{j}\right|=1$ and $\|w\|_{\infty} \leq 1$, we get $\operatorname{ubc}\left(y_{j} \mid X_{\infty}^{n}\right) \leq 3 \mathrm{gl}\left(X_{\infty}^{n}\right)$, completing the proof of (31). Now the conclusion follows from (31) and (d).

Since the space $X_{\infty}^{n}$ is translation invariant, statement (a) can be derived directly from (27), since $P$ is the orthogonal projection. On the other hand, 
it seems that condition (b) cannot be proved without a general argument of Lemma 6.

Observe that the John's ellipsoid on $X_{\infty}^{n}$ is actually the unit ball from $X_{2}^{n}$. This follows immediately from the uniqueness of the distance inner product, established in (c), and the fact that the John's ellipsoid actually determines this distance.

Actually, as stated in Remark 4, the gl-constant of an $n$-dimensional space $X_{n}$ satisfies $\operatorname{gl}\left(X_{n}\right) \leq \sup _{Y_{n}} \lambda\left(Y_{n}\right) \leq \sqrt{n}-c / \sqrt{n}$. This is easily seen by taking the 1-summing factorization of any operator $u: X_{n} \rightarrow l_{2}^{n}$.

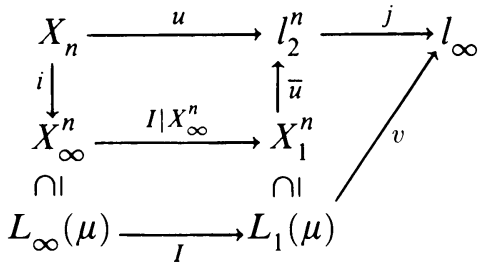

Here $\pi_{1}(I)=1$, and $\|v\|=\|\bar{u}\|=\pi_{1}(u)$. Projecting from $L_{1}(\mu)$ onto the subspace $X_{1}^{n}$ with $\|P\|=\lambda\left(X_{1}^{n}\right) \leq \sqrt{n}-c / \sqrt{n}$ yields an $L_{1}(\mu)$-factorization of $u: X_{n} \rightarrow l_{2}^{n}$. Thus

$$
\operatorname{gl}\left(X_{n}\right)=\sup \left\{\gamma_{1}(u) / \pi_{1}(u) \mid u \neq 0\right\} \leq \lambda\left(X_{1}^{n}\right) \leq \sqrt{n}-c / \sqrt{n} .
$$

Remark 6. It is not difficult to see that the type 2 constants of the spaces $X_{\infty}^{n}$ of Proposition 5 are of order $\sqrt{\ln n}$ and the (Gaussian) cotype 2 constants are of order $\sqrt{n / \ln n}$. An invariant closely related to the Gordon-Lewis constant was introduced in [PS] and called the Ké-constant. An argument somewhat similar to Proposition 5(f) shows that the Ké-constants of $X_{\infty}^{n}$ are between $\sqrt{n} / 3$ and $\sqrt{n}$.

To our best knowledge, the spaces $X_{\infty}^{n}$ provide the first deterministic construction of spaces with the gl-constants of maximal order. Examples known before consist of random subspaces of $l_{\infty}^{n}$, with $N$ proportional to $n$ (cf., e.g., [FJ], also [TJ, §34]).

\section{REFERENCES}

[FJ] T. Figiel and W. B. Johnson, Large subspaces of $l_{\infty}^{n}$ and estimates of the Gordon-Lewis constant, Israel J. Math. 37 (1980), 92-112.

[HR] H. Halberstam and K. F. Roth, Sequences, Springer-Verlag, New York, 1983.

[GG] D. J. H. Garling and Y. Gordon, Relations between some constants associated with finite dimensional Banach spaces, Israel J. Math. 9 (1971), 346-361.

[KaL] G. A. Kabatyanskii and V. I. Levenštein, Bounds for packings on a sphere and in a sphere, Problems Inform. Transmission 14 (1978), 1-17.

[KaS] M. J. Kadets and M. G. Snobar, Certain functionals on the Minkowski compactum, Math. Notes 10 (1971), 694-696 (Russian).

[Ke] A. M. Kerdock, A class of low-rate nonlinear binary codes, Inform. and Control 20 (1972), 182-187.

[Kö] H. König, Spaces with large projection constants, Israel J. Math. 50 (1985), 181-188. 
[KöL] H. König and D. R. Lewis, A strict inequality for projection constants, J. Funct. Anal. 73 (1987), 328-332.

[KöLL] H. König, D. R. Lewis, and P. K. Lin, Finite dimensional projection constants, Studia Math. 75 (1983), 341-358.

[Lev] V. I. Levenštein, Bounds on the maximal cardinality of a code with bounded modules of the inner product, Soviet Math. Dokl. 2 (1982), 526-531.

[Lew1] D. R. Lewis, Finite-dimensional subspaces of $L_{p}$, Studia Math. 63 (1978), 207-212.

[Lew2] _ An upper bound for the projection constant, Proc. Amer. Math. Soc. 103 (1988), $1157-1160$.

[LT] J. Lindenstrauss and L. Tzafriri, Classical Banach spaces I, Springer-Verlag, New York, 1977.

[M] C. Müller, Spherical harmonics, Lecture Notes in Math., vol. 17, Springer-Verlag, 1966.

[PS] A. Pelczynski and C. Schütt, Factoring the natural injection $i^{(n)}: L_{n}^{\infty} \rightarrow L_{n}^{1}$ through finite dimensional Banach spaces and geometry of finite dimensional unitary ideals, J. Math. Anal. Appl. 7 (1981), 653-683.

[Pis] G. Pisier, Some results on Banach spaces without unconditional structure, Compositio Math. 37 (1978), 3-19.

[R] W. Rudin, Trigonometric series with gaps, J. Math. Mech. 9 (1960), 203-227.

[TJ] N. Tomczak-Jaegermann, Banach-Mazur distances and finite-dimensional operator ideals, Pitman Monographs and Surveys in Pure and Appl. Mathematics, 38, Longman Scientific and Technical, Harlow and Wiley, New York, 1989.

Mathematisches Seminar, University of Kiel, 2300 Kiel, German Federal Republic

Department of Mathematics, University of Alberta, Edmonton, Alberta, Canada T6G 2G1 